HNO 2022 $\cdot 70: 206-213$

https://doi.org/10.1007/s00106-021-01101-5

Angenommen: 24. Juni 2021

Online publiziert: 3 . September 2021

(c) Der/die Autor(en) 2021

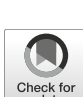

Check for
updates

\title{
Endoskopische Vermessung von Nasenseptumdefekten
}

\author{
Jean-Claude Rosenthal' - Eric L. Wisotzky ${ }^{1,3}$. Carsten Matuschek ${ }^{2} \cdot$ Melanie Hobl $^{4}$. \\ Anna Hilsmann ${ }^{1}$ Peter Eisert ${ }^{1,3}$. Florian C. Uecker ${ }^{4}$ \\ 'Vision and Imaging Technologies, Fraunhofer Heinrich-Hertz-Institut HHI, Berlin, Deutschland \\ ${ }^{2}$ MKG-Klinik, Charité - Universitätsmedizin Berlin, Berlin, Deutschland \\ ${ }^{3}$ Visual Computing, Humboldt Universität zu Berlin, Berlin, Deutschland \\ ${ }^{4}$ HNO-Klinik, Charité - Universitätsmedizin Berlin, Berlin, Deutschland
}

\section{Zusammenfassung}

Hintergrund: Die vielfältigen unangenehmen Symptome von Nasenseptumdefekten (NSD) führen zu einer deutlichen Einschränkung der Lebensqualität. NSD können mittels patientenspezifischer Implantate oder durch eine Op. verschlossen werden. Implantate werden dabei durch Silikonabformungen unter Vollnarkose, ggf. in lokaler Betäubung, oder anhand von 3D-Modellen aus computertomographischen (CT-)Daten erstellt. Nachteile für die Patientensicherheit sind ein erhöhtes Morbiditätsrisiko oder die Strahlenbelastung.

Methodik: Für die Hals-, Nasen- und Ohren-(HNO)-Chirurgie wird ein schonender Ansatz zur Behandlung von NSD mit einer neuen bildbasierten, kontakt- und strahlungsfreien Messmethode unter Nutzung eines Stereoendoskops vorgestellt. Das Verfahren beruht ausschließlich auf Bilddaten und nutzt echtzeitfähige Bildverarbeitungsalgorithmen zur Berechnung von 3D-Informationen. Es ist beliebig oft wiederholbar und wurde bereits erfolgreich in der robotergestützten Chirurgie und in der OP-Mikroskopie eingesetzt. Daher wurde diese Methode für die Nasenchirurgie erweitert, für die es zusätzliche anatomische und stereoskopische Herausforderungen gibt.

Ergebnisse: Nach Auswertung von 3 relevanten Messgrößen (NSD-Ausdehnung: axial, koronal und Umfang) von 6 Patienten und Vergleich der Ergebnisse von 2 Stereoendoskopen mit vorhandenen CT-Daten zeigte sich: Die bildbasierten Messergebnisse können vergleichbare Genauigkeiten wie CT-Daten erzielen. Bei einem Patienten wurden die Daten nur teilweise ausgewertet, da der NSD größer als das endoskopische Sichtfeld war.

Schlussfolgerung: Aufbauend auf den sehr guten Messwerten wird ein Therapieverfahren skizziert, welches die Herstellung von patientenspezifischen NSD-Implantaten auf Basis endoskopischer Daten ermöglicht.

\section{Schlüsselwörter}

Endoskopie - Nasenerkrankungen · Bildgesteuerte Therapie - Rekonstruktive chirurgische Verfahren · 3D-Rekonstruktion

Die englische Version dieses Beitrags ist unter https://doi.org/10.1007/s00106-021-01102-4 zu finden.

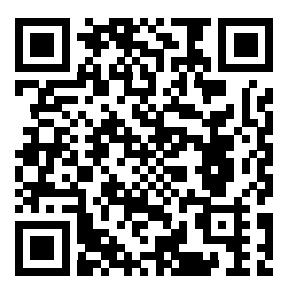

QR-Code scannen \& Beitrag online lesen
In der HNO-Chirurgie und speziell in der Rhinologie ist es von großer Bedeutung, dass die Nasenhöhle und deren Belüftungssystem störungsfrei und funktionsfähig sind. Eine Störung des Luftstroms stellt eine hohe Beeinträchtigung der Lebensqualität des Patienten dar. Dies trifft insbesondere für das Krankheitsbild der Nasenseptumdefekte (NSD) zu.
Bei einem Nasenseptumdefekt (NSD) wird die Atmung durch den Kontinuitätsdefekt im knorpeligen oder knöchernen Anteil der Nasenscheidewand beeinträchtigt, wobei die mukoperichondrale oder mukoperiostale Auskleidung fehlt. In • Abb. 1a sind die anatomischen Details an einem Nasspräparat im Querschnitt und ein Überblick über die Lage der Nasenscheidewand erfasst. Die - Abb. 1b zeigt eine Perforation unter endoskopischer Sicht. 

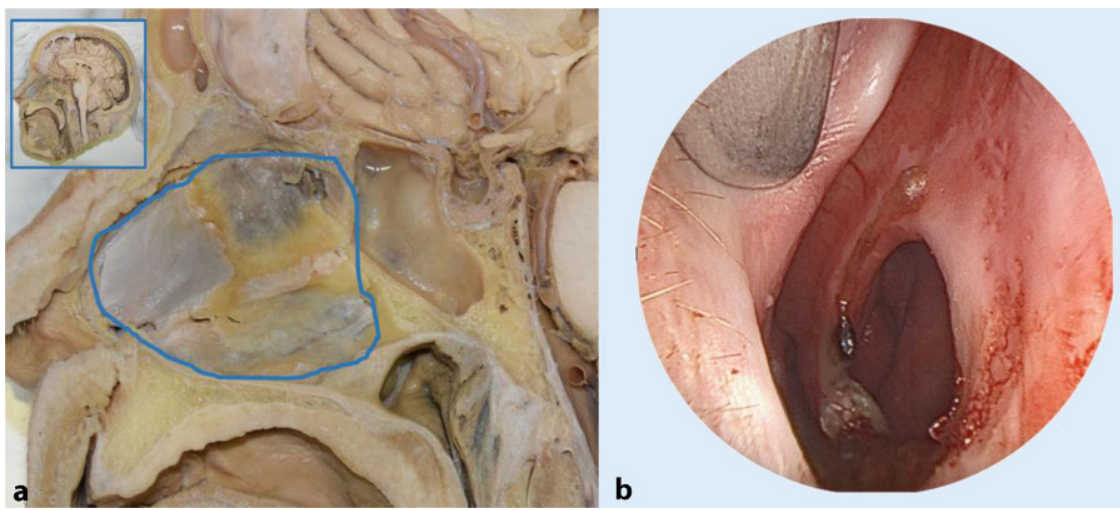

Abb. 1 ॥ Darstellung der Nasenscheidewandanatomie. a Nasspräparat - Querschnitt. b Endoskopische Ansicht des Nasenseptumdefekts (NSD)

Ein NSD kann zu vielfältigen und unangenehmen Symptomen führen, wie eingeschränkte Atmung, Nasenbluten, trockene bzw. reflektorisch zu feuchte Nase sowie Schlafstörungen. Daher ist ein Verschluss des Septums angezeigt, um einen konstanten nasalen Luftstrom wiederherzustellen. Aktuell gibt es 2 therapeutische Strategien zur Behandlung von NSD-Symptomen: (1) den Einsatz von pflegenden Sprays und Salben zur Linderung der Beschwerden und (2) kausal der chirurgische bzw. nichtchirurgische Verschluss der Perforation. Die Perforation kann temporär mit einsetzbaren Nasenseptumbuttons oder operativ dauerhaft mit Eigengewebe verschlossen werden. Die Implantate werden anhand von analogen und digitalen Modellen gefertigt. Die Modelle werden entweder aus Silikonabformungen unter Vollnarkose oder in lokaler Betäubung oder aus computertomographischen(CT-)Daten generiert. Beide Methoden haben jedoch einen Einfluss auf die Patientensicherheit, da sie das Morbiditätsrisiko erhöhen oder die Strahlenhygiene verletzen.

\section{Zielsetzung}

Die vorliegende Arbeit befasst sich mit der nichtchirurgischen Behandlung von NSD mittels eines temporären Verschlusses. Dazu stellen die Autor:innen eine neue bildbasierte, kontakt- und strahlungsfreie Messmethode unter Verwendung eines Stereoendoskops vor. Es werden die 3DEndoskop-Messergebnisse mit den CTDaten als Goldstandard verglichen. Das
Ergebnis dieser Arbeit soll einen Beitrag dazu liefern, inwiefern endoskopische/ bildbasierte Messverfahren etablierte therapeutische Ansätze ersetzen/ergänzen können, um zukünftig das Patientenrisiko zu reduzieren.

\section{Klinische Beschreibung von NSD}

Der schichtübergreifende Defekt der Nasenscheidewand ( $\bullet \mathbf{A b b} .1 \mathbf{b})$ verändert die Geschwindigkeit und den Volumendurchfluss in der Nasenhöhle. Diese Faktoren sind bekanntermaßen entscheidend für nasale Funktionen wie Geruchssinn, Filtration, Erwärmung und Befeuchtung der eingeatmeten Luft [10, 26]. Posteriore und kleinere Perforationen verursachen aufgrund der weiterbestehenden befeuchtenden Wirkung der Nasenschleimhaut und der Nasenmuscheln tendenziell weniger Symptome [2, 23]. Die Prävalenz eines NSD liegt zwischen 0,9 und 2,1 \% in der Bevölkerung und nach Septumplastik sogar bei bis zu $25 \%[7,13,15,24]$. Es gibt dabei keinen bekannten Zusammenhang zwischen einem Septumdefekt und Faktoren wie Alter, Geschlecht oder geografischer Lage. Die Ursachen für NSD sind vielfältig; verschiedene Ätiologien wie traumatische Perforation, berufliche Exposition, persönliche Gewohnheiten, Drogenmissbrauch, topische oder systemische Medikation sowie bestimmte Autoimmunerkrankungen sind bekannt $[4,8,16,25,28,32]$.

\section{NSD-Therapie heute}

Grundsätzlich sollte ein NSD operativ verschlossen werden, wenn dessen Größe es zulässt und der Allgemeinzustand des Patienten nicht dagegen spricht $[20,21]$. Eine Versorgung mittels industriell gefertigtem oder individuell angepasstem Button ist immer eine Kompromisslösung. Die nichtchirurgische NSD-Therapie durch temporären Verschluss erfolgt mit Implantaten, sog. Nasenscheidewandbuttons. Diese Implantate können einteilig sowie zweiteilig mit Magneten hergestellt werden ( $\bullet$ Abb. $2 c, \mathbf{g}$ ). Aus den CTDaten wird mittels einer Computer-Aided-Design(CAD)-Software ein 3D-Modell generiert ( $\bullet$ Abb. 2a). Aus den virtuellen Daten wird anschließend ein physisches Modell mit Computer-Aided Manufacturing (CAM) in einem 3D-Drucker hergestellt (- Abb. 2b). Anhand dieses gedruckten Modells wird der zweiteilige Septumbutton mit Magneten hergestellt ( $\square$ Abb. $2 c)$. Der fertige zweiteilige Septumbutton muss spannungsfrei und bündig am Modell anliegen (- Abb. 2d). Analoge Abformungen ( $\mathbf{A b b}$.2e) bieten eine weitere Möglichkeit zur Darstellung einer NSD. Aus diesen Abformungen wird ein Gipsmodell hergestellt ( $\bullet$ Abb. 2f). Aufgrund der Größenlimitierung der mit Silikon abzuformenden Perforation von etwa $3 \mathrm{~cm}^{2}$ wird i. d. R. ein einteiliges Implantat für den NSD

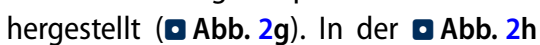
ist ein Septumbutton im analogen Gipsmodell dargestellt, auch hier müssen die Ränder spannungsfrei und bündig anliegen. Industriell konfektionierte Implantate sind nahezu obsolet aufgrund ihrer schlechten Passung und der damit verbundenen Krustenbildung. Diese kann unter den Kofaktoren lokale Infektion, Manipulationen durch den Patienten oder Arzt im Rahmen der Reinigung und durch Schleimhautnekrose aufgrund unzureichender Pflege zu einer Vergrößerung der Perforation führen. Zudem kann es bei ungenau passenden Buttons zu Druckstellen ebenfalls zu Schleimhautnekrose und konsekutiver Perforationsvergrößerung kommen. Der Septumbutton wird nach Abschwellung der Schleimhäute unter Anwendung eines topischen Lokalanästhetikums durch das Nasenloch in den entsprechenden NSD eingesetzt. Zweiteilige Septumbuttons werden mittels Magneten oder Druckknöpfen durch den Septumdefekt miteinander verbunden. 


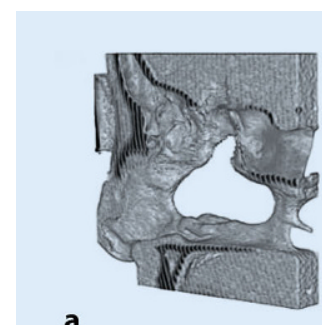

a

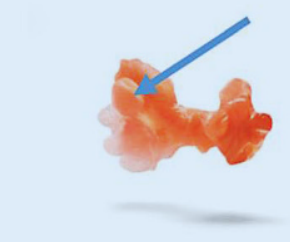

e

3-D Rohdaten b

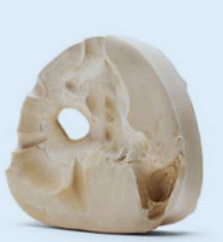

f

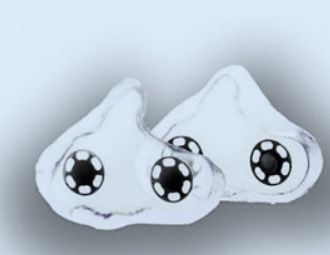

c

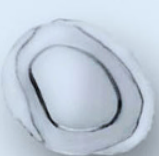

g

Implantat

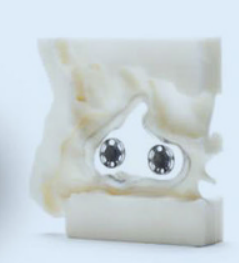

d

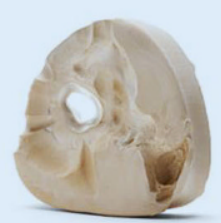

h

Revision
Abb. $2 \triangleleft$ Herstellungsprozess für Nasenscheidewandbuttons. a-d Computertomographie(CT)Scans, e-h Silikonabformung
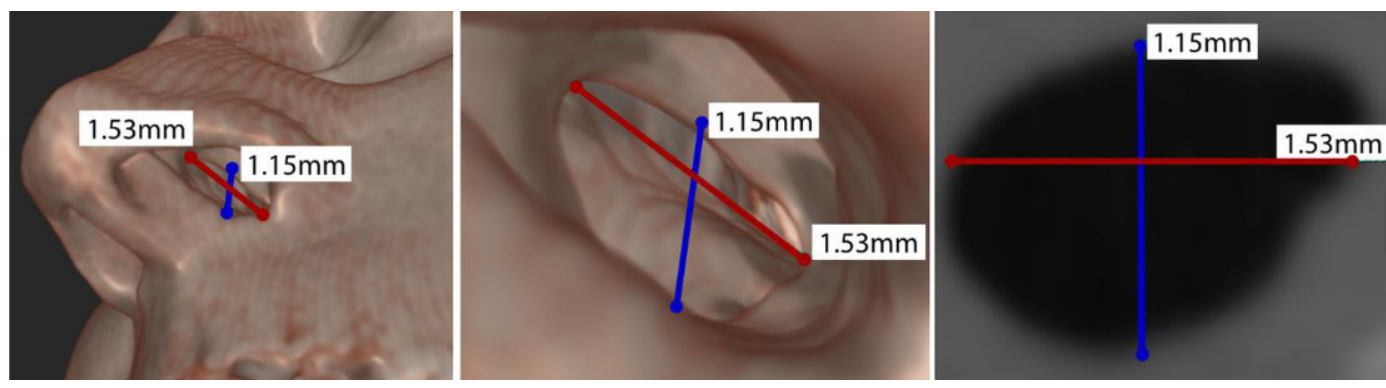

Abb. $3<$ Computertomographie(CT)-Scan. Bestimmung der Ausdehnung des Nasenseptumdefekts (NSD) in horizontaler und vertikaler Richtung

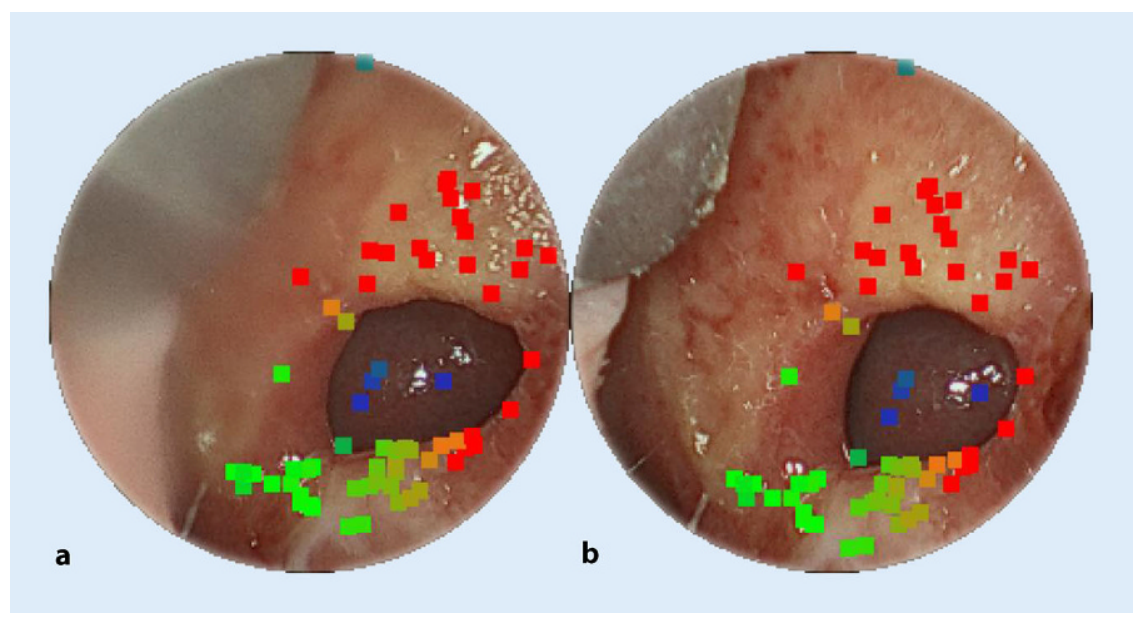

Abb. 4 \ Korrespondierende Merkmalspunkte in (a) linker (b) rechter Stereoansicht des Nasenseptumdefekts (NSD) für die rechte Nasenhaupthöhle

Alle Methoden haben jedoch Nachteile aus Patientensicht: Konfektionierte Implantate sind nicht patientenspezifisch und haben eine schlechte Passung, die zu Krustenbildungen führen kann. Bei sehr großen Defekten sind Silikonabformungen aufgrund fehlender Auflagepunkte größenlimitiert, außerdem erfordern sie eine Betäubung und können zu Verletzun- gen führen (erhöhtes Morbiditätsrisiko). CT-Daten stehen im Vergleich dazu im Konflikt mit der Strahlenhygiene. Jedes Verfahren erfordert unabhängig davon eine anatomische Vermessung der meist nicht kreisförmigen Perforation. Typische Perforationen können von wenigen Submillimetern bis zu mehreren Zentimetern reichen. Bei der Größenbestimmung ist es zudem wichtig, die vertikale als auch die horizontale Länge der Perforation zu messen (-Abb. 3). Insbesondere die vertikale Perforationshöhe spielt eine Schlüsselrolle für den Therapieerfolg, da sie unmittelbare Auswirkungen auf die Spannung zwischen Nasenboden und Nasenrücken hat $[4,9]$. Zudem ist eine spätere chirurgische Behandlung nicht ausgeschlossen. Für chirurgische Behandlungen sind mehrere Techniken mit unterschiedlichen Erfolgsraten beschrieben. Sie beruhen auf 2 Grundprinzipien:

- der Verwendung von Schleimhautlappen und

- dem Einsetzen eines Interpositionselements zwischen den beiden Schleimhautoberflächen [6, 14, 20-23].

Es muss jedoch immer eine Abwägung für die jeweilige therapeutische Methode erfolgen, da insbesondere Patienten mit hochgradigen Septumdeviationen unter aktiven Blutungen leiden können. Hier sehen die Autor:innen in der akuten Blutungssituation eine relative Kontraindikation zur Sofortversorgung. Darüber hinaus 
Tab. 1 Übersicht über die Studiengruppe

\begin{tabular}{|l|l|l|l|l|}
\hline Nr. & Geschlecht & $\begin{array}{l}\text { Alter } \\
\text { (Jahre) }\end{array}$ & $\begin{array}{l}\text { Dauer } \\
\text { (Monate) }\end{array}$ & Grund für Septumdefekt \\
\hline 1 & Männlich & 36 & 38 & Drogenmissbrauch \\
\hline 2 & Männlich & 56 & 480 & Spätfolgen/Komplikation Septumplastik \\
\hline 3 & Weiblich & 35 & 52 & Granulomatose mit Polyangiitis/M. Wegener \\
\hline 4 & Weiblich & 52 & 84 & Spätfolgen/Komplikation Septorhinoplastik \\
\hline 5 & Männlich & 81 & 264 & Trauma \\
\hline 6 & Männlich & 33 & 26 & Spätfolgen/Komplikation Septumplastik \\
\hline
\end{tabular}

müssen bei Patienten mit aktiver lokaler Infektion oder unter Verwendung von intranasal zu applizierenden Medikamenten die Vor- und Nachteile der jeweiligen Versorgung kritisch überdacht werden [4, 12, 17].

\section{Endoskopische 3D-Vermessung in der Chirurgie}

Bildbasierte strahlungsfreie 3D-Messverfahren wurden bereits in anderen chirurgischen Disziplinen verwendet, in denen die intraoperative Unterstützung von großem Interesse ist, u. a. in der robotergestützten Chirurgie, der 3D-Laparoskopie oder in der OP-Mikroskopie [1, 3, 11, 19, 30]. Im Vergleich zur Viszeralchirurgie und OP-Mikroskopie sind ein kleineres Sichtfeld sowie starke perspektivische Variationen durch extreme stereoskopische Blickwinkel die Hauptunterschiede für die Nasenchirurgie. Unter Berücksichtigung aller therapeutischen, technischen und chirurgischen Gegebenheiten besteht daher ein hoher Bedarf an der Entwicklung einer kontaktlosen und strahlungsfreien Messmethode zur Erstellung von patientenspezifischen anatomischen 3D-Implantaten bei gleichzeitiger Steigerung der Patientensicherheit und Implantatqualität.

\section{Methodik}

Die Autor:innen verwenden ein Stereoendoskop für die Aufnahme des NSD, um ein digitales 3D-Anatomiemodell zu berechnen. Dazu werden korrespondierende Merkmalspunkte bestimmt, die die gleichen anatomischen Landmarken für beide Stereoansichten beschreiben (• Abb. 4). Daraus lässt sich die sog. Stereoparallaxe oder binokulare Disparität berechnen. Auf diese Weise können die Autor:innen bildbasierte Messungen mittels Triangulation bei bekannten Kameraeigenschaften (u.a. Brennweite) durchführen, um relevante 3D-Informationen aus den endoskopischen Bilddaten zu rekonstruieren.

\section{Studiengruppe}

Diese Methode wurde mit der Zustimmung von 6 Patienten unterschiedlichen Geschlechts, Alters und mit unterschiedlichen Ursachen für den NSD evaluiert (- Tab. 1). Die Studiengruppe bestand aus 3 jungen Erwachsenen (33-36 Jahre alt), 2 mittleren Alters (52-56 Jahre) und einer älteren Person (81 Jahre), die unterschiedliche Ätiologien und eine unterschiedliche Dauer des Septumdefekts seit der Erstdiagnose aufwiesen.

\section{Bildgebung und Auswertung}

Die Stereobilder wurden mit 2 verschiedenen Stereoendoskopen aufgenommen. Das erste Endoskop stammt von der Fa. XION MEDICAL GmbH, Berlin, und wurde speziell für HNO-Eingriffe konzipiert. Das zweite Endoskop ist ein 3D-Laparoskop der Fa. Schölly Fiberoptic $\mathrm{GmbH}$, Denzlingen. Die Daten der Patienten Nr. 2, 3 und 5 wurden mit dem HNO-Endoskop (4 mm Durchmesser, $0^{\circ}$-Optik, $80^{\circ}$ Öffnungswinkel, $60 \mathrm{fps} \mathrm{(,frames} \mathrm{per} \mathrm{second",}$ Bilder pro Sekunde), keine Zoommöglichkeit, Fokus veränderbar) aufgenommen, während die Daten der Patienten Nr. 1, 4 und 6 mit dem 3D-Laparoskop $(10 \mathrm{~mm}$, $0^{\circ}$-Optik, $72^{\circ}$ Öffnungswinkel, $25 \mathrm{fps}$, keine Zoommöglichkeit, Fokus nicht veränderbar) aufgenommen wurden. Beide endoskopischen Systeme haben eine Bildauflösung von $1920 \times 1080$ px (Pixel) pro Stereokanal. Das Verfahren gliedert sich in mehrere Schritte und stützt sich auf echtzeitfähige Bildverarbeitungsalgorithmen [29]. Zunächst muss das 3D-Endoskop ka- libriert werden, da die Messanwendungen und die Stereobildverarbeitung fest miteinander verknüpft sind. Die Kalibrierung eines optischen Bildverarbeitungssystems ist ein Offline-Vorverarbeitungsschritt, der die optischen Kameraparameter wie Brennweite, Stereobasis und Linsenverzerrungen berechnet. Für die Kalibrierdaten gilt, dass diese nur für eine feste Einstellung aus Zoom und Fokus Gültigkeit besitzen. Sollten die Vergrößerung oder die Fokuseben verändert werden, hat dies Einfluss auf die Messgenauigkeit. Dazu verwenden die Autor:innen eine geräteunabhängige Kalibrierung unter Einsatz eines schachbrettähnlichen Referenzkörpers [18, 31]. Darüber hinaus unterscheidet sich das hier eingesetzte Kalibrierverfahren von bekannten Methoden durch einen modellbasierten Ansatz mit Gradientenabstieg und Bildregistrierung zur Korrelation mit der Referenzebene [5]. Die komplette 3D-Rekonstruktions- und Vermessungskette besteht aus 3 Schritten:

- Stereorektifizierung,

- Stereoparallaxen-/Disparitätsschätzung und

- die metrische Vermessung der Szene.

Die Rektifizierung des Stereobilds erfolgt über die Erkennung von robusten Merkmalspunkten ([33]; - Abb. 4), um eine Homographie-Matrix abzuleiten, die garantiert, dass die Stereobilder frei von vertikalen Disparitäten sind. Anschließend wird eine Disparitätsschätzung [27] mit den korrigierten Stereobildpaaren durchgeführt. Die subpixelgenaue Disparitätsschätzung berücksichtigt zeitlichräumliche Abhängigkeiten zwischen den zu bestimmenden Korrespondenzpunkten. Die Korrespondenzen werden dabei lokal ermittelt. Die iterative und unabhängige Verteilung der Korrespondenzen garantiert, dass die gesamte Szene unter Beibehaltung der zeitlich-räumlichen Konsistenz global aktualisiert wird. Nach einer kurzen Initialisierungsphase von 20 Stereobildpaaren erhält man eine komplette Repräsentation der Szene als Disparitätskarte. Abschließend rekonstruieren die Autor:innen die NSD-Anatomie maßstabsgetreu aus den berechneten Disparitätskarten und den vorab bestimmten Kamerakalibrierdaten. Auf den rekonstruierten Endoskopiedaten werden dann die 


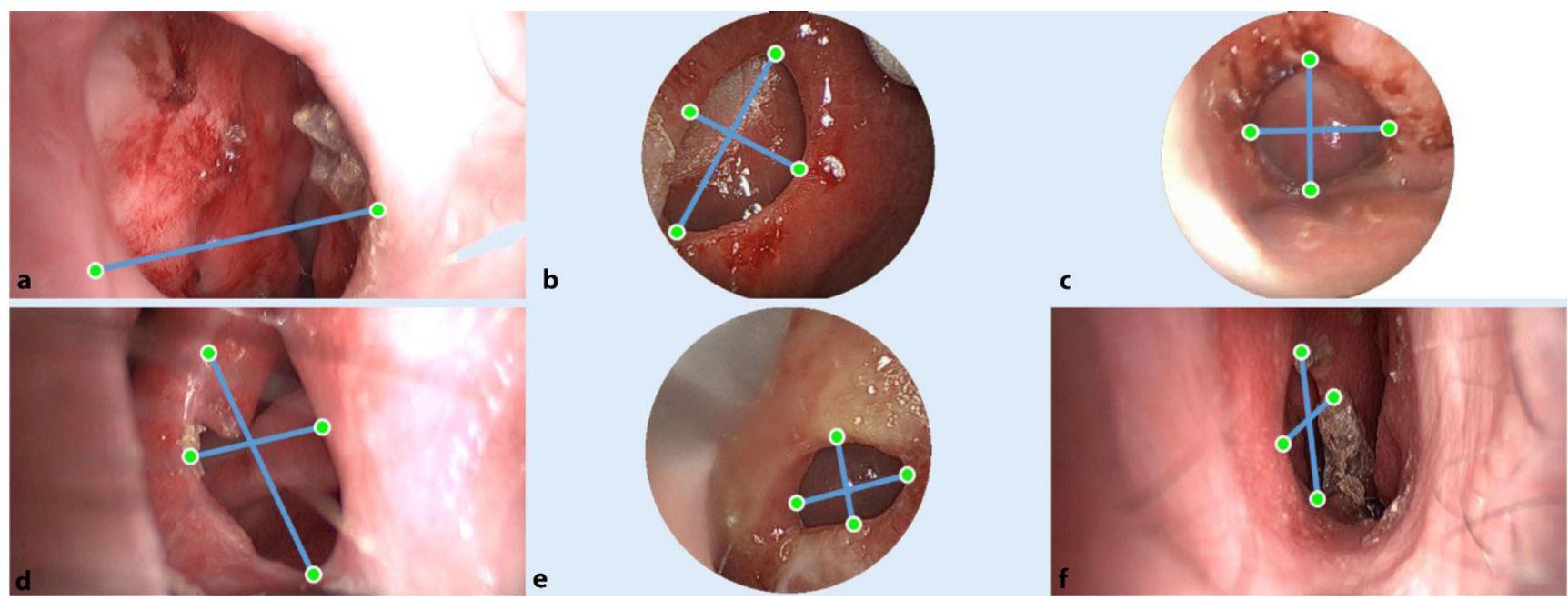

Abb. 5 ॥ Übersicht der Nasenseptumdefekte (NSD) der Studiengruppe aus - Tab. 1 und Visualisierung der axialen und koronalen Messpunkte. Dazugehörige Messergebnisse in DTab. 2. (a) Patient 1, (b) Patient 2, (c) Patient 3, (d) Patient 4, (e) Patient 5 und (f) Patient 6

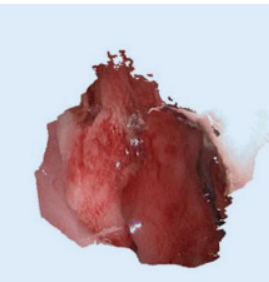

a

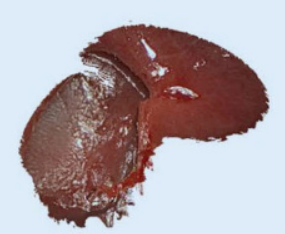

b

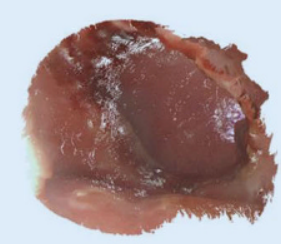

C

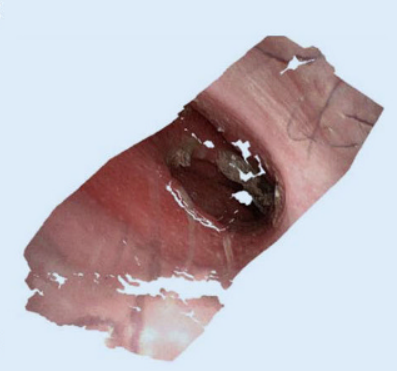

alle Patienten mit Ausnahme von Nr. 1 wurde die axiale und koronale Achse sowie der Umfang der Perforation bestimmt. Die relativen Abweichungen liegen innerhalb der geforderten Messgenauigkeit von $5 \%$. Der Mittelwert aller Absolutdifferenzen beträgt $0,28 \mathrm{~mm}$ mit einer Standardabweichung (SD) von $0,146 \mathrm{~mm}$ für die axiale Achse und $0,16 \mathrm{~mm}$ mit einer SD von $0,09 \mathrm{~mm}$ für die koronale Achse sowie $0,904 \mathrm{~mm}$ mit einer SD von $0,769 \mathrm{~mm}$ für den Umfang. Bei allen durchgeführten Messungen betragen die Absolutfehler weniger als $0,5 \mathrm{~mm}$ und können somit vergleichbare Messwerte wie CT-Daten liefern. Es gibt keine signifikanten Unterschiede zwischen den beiden endoskopischen Systemen in Bezug auf Genauigkeit und Messunsicherheit. Die Messgenauigkeit bestimmt sich hier aus den Parametern des Stereosystems: Brennweite, Stereobasis, Bildauflösung, Pixelgröße sowie aus dem entferntesten Punkt, der in die Messung eingegangen ist. Patient Nr. 1 bedarf einer weiteren Erklärung ( $\bullet$ Abb. 5a), da die vorgeschlagene Methode keine sinnvolle axiale Messung und daher keine Bestimmung des Umfangs ermöglichte. Hierfür gibt es 2 Hauptgründe. Zum einem war es nicht möglich, die Perforationsausdehnung mit einer einzigen Aufnahme zu erfassen, da das endoskopische Sichtfeld aufgrund der extremen Größe der Perforation von $4,50 \mathrm{~cm}$ in der axialen Dimension überschritten wurde. 
Tab. 2 Evaluierung und Vergleich der Messergebnisse für Stereoendoskop- und Computertomographie(CT)-Daten

\begin{tabular}{|l|l|l|l|l|l|l|l|l|l|l|l|l|}
\hline & \multicolumn{2}{|l}{ Stereoendoskop-Messung $(\mathbf{m m})$} & \multicolumn{2}{l|}{ CT-Messung (mm) } & \multicolumn{3}{l|}{ Relative Messabweichung (\%) } \\
\hline Nr. & Axial & Koronal & Umfang & Messunsicherheit & Axial & Koronal & Umfang & Voxelgröße & Axial & Koronal & Umfang \\
\hline 1 & - & 18,8 & - & 0,3 & 45,0 & 19,1 & 12,30 & 0,5 & - & 1,57 \\
\hline 2 & 9,60 & 7,30 & 27,96 & 0,2 & 9,20 & 7,40 & 27,30 & 0,625 & 4,35 & 1,35 \\
\hline 3 & 15,5 & 11,8 & 44,39 & 0,2 & 15,3 & 11,5 & 44,70 & 0,625 & 1,31 & 2,61 & 0,69 \\
\hline 4 & 11,3 & 15,1 & 43,50 & 0,2 & 11,1 & 15,0 & 42,30 & 0,625 & 1,80 & 0,67 & 2,84 \\
\hline 5 & 7,40 & 5,60 & 20,20 & 0,4 & 7,50 & 5,40 & 20,30 & 0,625 & 1,33 & 3,70 & 0,49 \\
\hline 6 & 24,4 & 9,9 & 63,05 & 0,7 & 24,9 & 10,0 & 60,80 & 0,625 & 2,01 & 1,00 & 3,70 \\
\hline
\end{tabular}

Außerdem konnten wichtige Bereiche aufgrund der stereoskopischen Verdeckung durch einen spitzen Blickwinkel nicht berechnet werden. Zum anderen wurde die Bildqualität durch die Bewegungsunschärfe der Kamera und durch atmungsbedingte Kondensation auf der Endoskopoptik negativ beeinflusst. Bewegungsunschärfe tritt bei beiden Objektiven auf, während Kondensation auch nur bei einem Objektiv auftreten kann. In beiden Fällen nimmt die Bildqualität stark $a b$, und es ist nicht mehr möglich, zuverlässige Korrespondenzen zu finden, um daraus die erforderlichen Tiefeninformationen zu berechnen.

Neben den rein bildbasierten relevanten Vermessungsparametern wurden auch die dazugehörigen 3D-Punktwolken (• Abb. 6) vollständig berechnet, um eine gezielte 3D-Visualisierung zur quantitativen Bewertung der Anatomie zu ermöglichen. Das hier eingesetzte bildbasierte 3DRekonstruktionsverfahren wurde bereits in der robotisch assistierten Chirurgie an 3D-Referenzanatomien von Schweinekadavern evaluiert. Es ist echtzeitfähig und liefert sehr genaue Messergebnisse mit sehr niedrigen Fehlerraten im Soll/Ist-Vergleich zur bekannten Referenzgröße [1]. Die hier erzielten Ergebnisse bestätigen somit die generelle Leistungsfähigkeit des 3D-Rekonstruktionsverfahrens für eine weitere chirurgische Anwendung mit zusätzlichen Anforderungen an die Algorithmik, die sich aus der speziellen Nasenanatomie sowie aus den stereosperspektivischen Besonderheiten ergeben.

\section{Diskussion und Ausblick}

In der vorliegenden Arbeit haben die Autor:innen einen neuen bildbasierten Ansatz für die Vermessung von wichtigen Anatomieparametern bei NSD mit einem
3D-Endoskop vorgestellt und mit vorhandenen CT-Daten verglichen. Die Methode zeigt akkurate und präzise Messergebnisse mit geringen relativen Abweichungen im Submillimeterbereich zur Bestimmung der maßstabgetreuen Dimension des NSD in horizontaler und vertikaler Richtung sowie bei der Bestimmung des Perforationsumfangs. Bei der bildbasierten Methode kann der Schleimhautdefekt präzise erfasst werden, es erfolgt dabei im Gegensatz zur CT-basierten Methode aber keine optische Erfassung des Knorpeldefekts, welcher deutlich größer als der Schleimhautdefekt sein kann. Die Messungen erfolgen aktuell noch im 2-D-Bild. Grafische CT-Vermessungen haben den Vorteil, dass vorliegende Septumdeviationen sowie individuell angepasste Buttons besser erfasst bzw. integriert werden können. Dies gilt auch für die Erfassung der Dicke des Septums am anterioren und posterioren Perforationsrand. Es konnten jedoch bereits jetzt hochauflösende 3D-Punktwolken erzeugt werden, die eine quantitative wie qualitative Beurteilung der Patientenanatomie durch die Chirurg:innen erlauben und zudem eine Erfassung der zuvor genannten Herausforderungen im Vergleich zu CT-Vermessungen möglich erscheinen lassen.

Nächste Schritte untersuchen die praktische Umsetzbarkeit dieses für den Patienten schonenden Ansatzes, um die 2 gängigen nichtchirurgischen Methoden (CTScan, Silikonabformung) zur Behandlung von NSD zu ersetzen. Unter anderem muss das Verfahren so erweitert werden, dass für alle Perforationsgrößen die entscheidenden NSD-Parameter vermessen werden können. Dies gilt insbesondere für NSD, die größer als etwa $3 \mathrm{~cm}^{2}$ sind und bei denen die chirurgische Szene nicht mit einer endoskopischen Einzelaufnahme erfasst werden kann. Die Therapie größerer Sep- tumdefekte könnte mit dem hier erbrachten Nachweis von hochpräzisen Messergebnissen aus Einzelaufnahmen vielversprechend angegangen werden. Dazu sollen die Messungen und die Oberflächenrekonstruktion für beide Nasenlöcher zueinander mit sog. 3D-Mosaik- und SLAMVerfahren („,simultaneous localization and mapping") zu einem globalen 3D-Modell registriert und fusioniert werden, sodass dieses für den 3D-Druck des Implantats genutzt und problemlos in die bestehenden Arbeitsabläufe von Epithetiker:innen integriert werden kann.

Fazit für die Praxis

- Intraoperative bildbasierte Messverfahren mit 3D-Endoskopen erzielen vergleichbare Messwerte zu computertomographischen (CT-)Daten bei gleichzeitiger Vermeidung der Strahlenbelastung und sind beliebig oft im klinischem Verlauf wiederholbar.

- Endoskope sind - im Vergleich zu CT-Geräten - günstige Sensoren mit der Möglichkeit einer gezielten Erweiterung ihrer Funktionalität durch ergänzende Algorithmen der künstlichen Intelligenz (KI).

- Die 3D-Rekonstruktion aus endoskopischen Bilddaten bildet die Basis für zukünftige Augmented-Reality Anwendungen in allen chirurgischen Disziplinen.

- 3D-Rekonstruktionsverfahren bilden die generelle Basis für patientenspezifische Implantatlösungen in der HNO-Chirurgie. 


\section{Korrespondenzadresse}

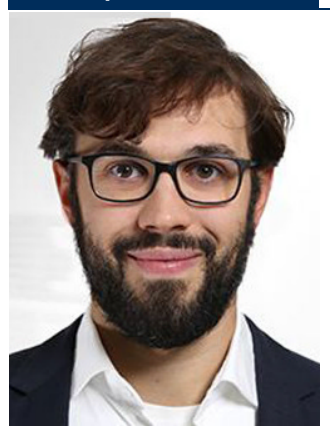

Dipl.-Inf. Jean-Claude Rosenthal

Vision and Imaging Technologies, Fraunhofer Heinrich-Hertz-Institut HHI

Einsteinufer 37, 10587 Berlin, Deutschland jean-claude.rosenthal@hhi.fraunhofer.de

Danksagung. Die Autor:innen danken den folgenden Personen und Partnern. Dr. rer. nat. Torsten Weiss, Institut für Funktionelle Anatomie, Charité Universitätsmedizin Berlin, für die Bereitstellung des Nasspräparats. Fa. ImFusion $\mathrm{GmbH}$, München, für die Bereitstellung ihrer Software für die Auswertung der CT-Daten. Fa. Schölly Fiberoptic GmbH, Denzlingen, für die Bereitstellung des 3D-Laparoskops.

Förderung. Diese Forschung wurde gefördert vom Bundesministerium für Bildung und Forschung (BMBF) unter dem Förderkennzeichen 16SV8061 (MultiARC).

Funding. Open Access funding enabled and organized by Projekt DEAL.

\section{Einhaltung ethischer Richtlinien}

Interessenkonflikt. J.-C. Rosenthal gibt folgenden Interessenkonflikt an: Forschungsförderung durch das Bundesministerium für Bildung und Forschung, Schölly Fiberoptic $\mathrm{GmbH}$ : Bereitstellung eines 3D-Endoskops. E.L. Wisotzky gibt folgenden Interessenkonflikt an: Forschungsförderung durch das Bundesministerium für Bildung und Forschung, ImFusion $\mathrm{GmbH}$ : Bereitstellung der Software "ImFusion Suite". M. Hobl, A. Hilsmann, P. Eisert und F.C. Uecker geben folgenden Interessenkonflikt an: Forschungsförderung durch das Bundesministerium für Bildung und Forschung. C. Matuschek gibt an, dass kein Interessenkonflikt besteht.

Die gesamte Forschung am Menschen entspricht allen relevanten nationalen Vorschriften, institutionellen Richtlinien und wurde in Übereinstimmung mit den Grundsätzen der Deklaration von Helsinki durchgeführt und von der Ethikkommission Charité - Universitätsmedizin Berlin unter der EA4/036/19 genehmigt.

Open Access. Dieser Artikel wird unter der Creative Commons Namensnennung 4.0 International Lizenz veröffentlicht, welche die Nutzung, Vervielfältigung, Bearbeitung, Verbreitung und Wiedergabe in jeglichem Medium und Format erlaubt, sofern Sie den/die ursprünglichen Autor(en) und die Quelle ordnungsge-

\section{Endoscopic measurement of nasal septum perforations. German version}

Background: Nasal septum perforations (NSP) have many uncomfortable symptoms for the patient and a highly negative impact on quality of life. NSPs are closed using patient-specific implants or surgery. Implants are created either under anesthesia using silicone impressions or using 3D models from CT data. Disadvantages for patient safety are the increased risk of morbidity or radiation exposure.

Materials and methods: In the context of otorhinolaryngologic surgery, we present a gentle approach to treating NSP with a new image-based, contactless, and radiationfree measurement method using a 3D endoscope. The method relies on image information only and makes use of real-time capable computer vision algorithms to compute 3D information. This endoscopic method can be repeated as often as desired in the clinical course and has already proven its accuracy and robustness for roboticassisted surgery (RAS) and surgical microscopy. We expand our method for nasal surgery, as there are additional spatial and stereoperspective challenges.

Results: After measuring 3 relevant parameters (NSP extension: axial, coronal, and NSP circumference) of 6 patients and comparing the results of 2 stereoendoscopes with $\mathrm{CT}$ data, it was shown that the image-based measurements can achieve comparable accuracies to CT data. One patient could be only partially evaluated because the NSP was larger than the endoscopic field of view.

Conclusion: Based on the very good measurements, we outline a therapeutic procedure which should enable the production of patient-specific NSP implants based on endoscopic data only.

\section{Keywords}

Endoscopy · Nose diseases · Image-guided therapy $\cdot$ Reconstructive surgical procedures $\cdot 3 \mathrm{D}$ reconstruction

mäß nennen, einen Link zur Creative Commons Lizenz beifügen und angeben, ob Änderungen vorgenommen wurden.

Die in diesem Artikel enthaltenen Bilder und sonstiges Drittmaterial unterliegen ebenfalls der genannten Creative Commons Lizenz, sofern sich aus der Abbildungslegende nichts anderes ergibt. Sofern das betreffende Material nicht unter der genannten Creative Commons Lizenz steht und die betreffende Handlung nicht nach gesetzlichen Vorschriften erlaubt ist, ist für die oben aufgeführten Weiterverwendungen des Materials die Einwilligung des jeweiligen Rechteinhabers einzuholen.

Weitere Details zur Lizenz entnehmen Sie bitte der Lizenzinformation auf http://creativecommons.org/ licenses/by/4.0/deed.de.

\section{Literatur}

1. Allan M, Mcleod J, Wang CC, Rosenthal JC, Fu KX, Zeffiro T, Xia W, Zhanshi Z, Luo H, Jia F, Zhang X, Li X, Sharan L, Kurmann T, Schmid S, Psychogyios D, Azizian M, Stoyanov D, Maier-Hein L, Speidel S (2021) Stereo correspondence and reconstruction of endoscopic data challenge https://arxiv.org/ abs $/ 2101.01133$

2. Bhattacharyya N (2007) Clinical symptomatology and paranasal sinus involvement with nasal septa perforation. Laryngoscope 117:691-694. https:// doi.org/10.1097/01.mlg.0000256455.01473.72

3. Bodenstedt S, Wagner M, Mayer B, Stemmer K, Kenngott H, Müller-Stich BP, Dillmann R, Spei- del S (2016) Image-based laparoscopic bowel measurement. Int J Comput Assist Radiol Surg 11:407-419

4. Downs BW, Sauder HM (2020) Septal perforation. StatPearls, Treasure Island (https://www.ncbi.nlm. nih.gov/books/NBK537208/)

5. Eisert $P$ (2002) Model-based camera calibration using analysis by synthesis techniques. International Workshop on Vision, Modeling, and Visualization, Berlin, Germany, S307-314

6. de Gabory L, Bareille R, Stoll D, Bordenave L, Fricain J-C (2010) Biphasic calcium phosphate to repair nasal septum: the first in vitro and in vivo study. Acta Biomater 6:909-919. https://doi.org/ 10.1016/j.actbio.2009.08.018

7. Gold M, Boyack I, Caputo N, Pearlman A (2017) Imaging prevalence of nasal septal perforation in an urban population. Clin Imaging 43:80-82. https://doi.org/10.1016/j.clinimag.2017.02.002

8. Kridel RWH (1999) Septal perforation repair. Otolaryngol Clin North Am 32:695-724. https:// doi.org/10.1016/S0030-6665(05)70165-1

9. Lee JY, Lee SH, Kim SC, Koh YW, Lee SW (2006) Usefulness of autologous cartilage and fibrin glue for the prevention of septal perforation during septal surgery: a preliminary report. Laryngoscope 116:934-937. https://doi.org/10.1097/01.MLG. 0000215174.47864.9A

10. Lindemann J, Keck T, Wiesmiller K, Sander B, Brambs H-J, Rettinger G, Pless D (2006) Nasal air temperature and airflow during respiration in numerical simulation based on multislice computed tomography scan. Am J Rhinol 20:219-223. https://doi.org/10.1177/194589240602000220

11. Maier-Hein L, Groch A, Bartoli A, Bodenstedt S, Boissonnat G, Chang PL, Clancy NT, Elson DS, 
Haase S, Heim E, Hornegger J, Jannin P, Kenngott $H$, Kilgus T, Müller-Stich B, Oladokun D, Röhl $S$, dos Santos TR, Schlemmer HP, Seitel A, Speidel S, Wagner M, Stoyanov D (2014) Comparative validation of single-shot optical techniques for laparoscopic 3-D surface reconstruction. IEEE Trans Med Imaging 33:1913-1930. https://doi.org/10. 1109/TMI.2014.2325607

12. Neumann A, Schneider M, Tholen C, Minovi A (2010) Inoperable Nasenseptumdefekte. HNO 58:364-370. https://doi.org/10.1007/s00106009-2072-3

13. Oberg D, Åkerlund A, Johansson L, Bende M (2003) Prevalence of nasal septal perforation: the Skövde population-based study. Rhinology 41:72-75

14. Parry JR, Minton TJ, Suryadevara AC, Halliday D (2008) The use of fibrin glue for fixation of acellular human dermal allograft in septal perforation repair. Am J Otolaryngol 29:417-422. https://doi. org/10.1016/j.amjoto.2007.11.001

15. Peacock MR (1981) Sub-mucous resection of the nasal septum. J Laryngol Otol 95:341-356. https:// doi.org/10.1017/s0022215100090812

16. Power DG, Kemeny NE (2011) Nasal septum perforation and bevacizumab. Med Oncol 28:89-93. https://doi.org/10.1007/s12032-010-9464-9

17. Romo T 3rd, Sclafani AP, Falk AN, Toffel PH (1999) A graduated approach to the repair of nasal septal perforations. Plast Reconstr Surg 103:66-75. https://doi.org/10.1097/00006534-19990100000012

18. Rosenthal JC, Gard N, Eisert P (2017) Kalibrierung stereoskopischer Systeme für medizinische Messaufgaben. Proceedings of the 16th Annual Conference of the German Society for Computer and Robotic Assisted Surgery (CURAC), Hannover, S161-163

19. Rosenthal J-C, Gard N, Schneider A, Eisert P (2018) Microscopic image-based determination of stapes prosthesis length. Conference Proceedings 32nd Int. Congress of Computer Assisted Radiology and Surgery, CARS, S59-60

20. Scheithauer M, Lindemann J, Sommer F, Wigand MCC (2021) Closure of nasal septal perforation. Laryngorhinootologie 100:224-232. https:// doi.org/10.1055/a-1309-0509

21. Schultz-Coulon H-J (2005) Three-layer repair of nasoseptal defects. Otolaryngol Head Neck Surg 132:213-218. https://doi.org/10.1016/j.otohns. 2004.09.066

22. Susman E (2007) Fibrin glue makes septal perforations easier to repair. https://www.enttoday.org/ article/fibrin-glue-makes-septal-perforationseasier-to-repair/.Zugegriffen:24. Febr. 2020

23. Tasca I, Compadretti GC (2006) Closure of nasal septal perforation via endonasal approach. Otolaryngol Head Neck Surg 135:922-927. https:// doi.org/10.1016/j.otohns.2006.04.017

24. Topal O, Celik SB, Erbek S, Erbek SS (2011) Risk of nasal septal perforation following septoplasty in patients with allergic rhinitis. Eur Arch Otorhinolaryngol 268:231-233. https://doi.org/10.1007/ s00405-010-1323-y

25. Traina TA, Norton L, Drucker K, Singh B (2006) Nasal septum perforation in a bevacizumabtreated patient with metastatic breast cancer. The Oncol 11:1070-1071. https://doi.org/10.1634/ theoncologist.11-10-1070

26. Uecker FC (2013) Hals-Nasen-Ohren-Heilkunde in Frage und Antwort, 2. Aufl. Urban \& Fischer in Elsevier, München

27. Waizenegger W, Feldmann I, Schreer O, Kauff P, Eisert $P$ (2016) Real-time 3D body reconstruction for immersive TV. 2016 IEEE International Conference on Image Processing (ICIP), S 360-364

28. Watson D, Barkdull G (2009) Surgical management of the septal perforation. Otolaryngol Clin North Am 42:483-493. https://doi.org/10.1016/j.otc. 2009.03.011

29. Wisotzky EL, Rosenthal J-C, Eisert P, Hilsmann A, Schmid F, Bauer M, Schneider A, Uecker FC (2019) Interactive and multimodal-based augmented reality for remote assistance using a digital surgical microscope. 2019 IEEE Conference on Virtual Reality and 3D User Interfaces (VR), S 1477-1484

30. Wisotzky EL, Rosenthal J-C, Wege U, Hilsmann A Eisert P, Uecker FC (2020) Surgical guidance for removal of cholesteatoma using a multispectral 3D-endoscope. Sensors 20:5334. https://doi.org/ $10.3390 / \mathrm{s} 20185334$

31. Zhang Z (1999) Flexible camera calibration by viewing a plane from unknown orientations. Proceedings of the Seventh IEEE International Conference on Computer Vision. Bd. 1, S666-673

32. Zhao K, Dalton P (2007) The way the wind blows: implications of modeling nasal airflow. Curr Allergy Asthma Rep 7:117-125. https://doi.org/10.1007/ s11882-007-0009-z

33. Zilly F, Riechert C, Eisert P, Kauff P (2011) Semantic kernels binarized-a feature descriptor for fast and robust matching. Visual Media Production (CVMP) 2011 Conference for IEEE, S39-48

\section{emeditorial
Manager}

\section{Hilfestellungen für den Editorial Manager}

Das Einreichungs- und Begutachtungssystem Ihrer Zeitschrift

Sowohl für die ganz alltäglichen Fragen in der Handhabung des Editorial Managers als auch für spezielle Problematiken finden Sie auf www.springermedizin.de/editorialmanager eine Vielzahl an Handreichungen, die Ihnen die Arbeit als Gutachter*in, Autor*in oder Herausgeber*in erleichtern.

Über Videos, einseitige Schritt-für-SchrittAnleitungen oder ein umfangreiches Manual werden Sie durch die einzelnen Punkte geführt, wie:

- Wie reiche ich ein Manuskript ein?

- Wie finde ich passende Gutachter*innen?

- Wie lade ich Gutachter*innen ein?

- Wie nehme ich ein Gutachten an bzw. lehne es ab?

- Wo erkenne ich, in welchem Status ein Mansukript ist?

- Wie ändere ich meine persönlichen Informationen?

- Wo kann ich meinen Urlaub eintragen?

Zugang auch über QR-Code:

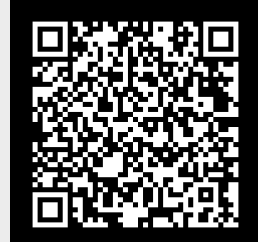

